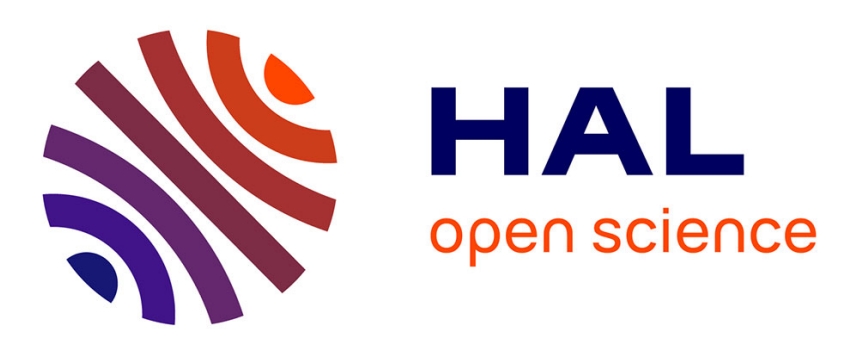

\title{
Simulation par éléments finis des procédés par infusion de résine
}

Guillaume Pacquaut, Julien Bruchon, Nicolas Moulin, Sylvain Drapier, Lara

Abouorm

\section{- To cite this version:}

Guillaume Pacquaut, Julien Bruchon, Nicolas Moulin, Sylvain Drapier, Lara Abouorm. Simulation par éléments finis des procédés par infusion de résine. 10e colloque national en calcul des structures, May 2011, Giens, France. pp.Clé USB. hal-00592736

\section{HAL Id: hal-00592736 https://hal.science/hal-00592736}

Submitted on 3 May 2011

HAL is a multi-disciplinary open access archive for the deposit and dissemination of scientific research documents, whether they are published or not. The documents may come from teaching and research institutions in France or abroad, or from public or private research centers.
L'archive ouverte pluridisciplinaire HAL, est destinée au dépôt et à la diffusion de documents scientifiques de niveau recherche, publiés ou non, émanant des établissements d'enseignement et de recherche français ou étrangers, des laboratoires publics ou privés. 


\title{
Simulation par éléments finis des procédés par infusion de résine
}

\author{
G. Pacquaut, J. Bruchon, N. Moulin, S. Drapier, L. Abou Orm
}

SMS, Ecole des Mines de Saint-Étienne, France, \{bruchon@emse.fr\}

\begin{abstract}
Résumé - Cet article expose un modèle numérique permettant de simuler par éléments finis les procédés par infusion de résine au travers de renforts fibreux. Ce modèle nécessite le couplage entre un écoulement dans un milieu poreux (Darcy), non poreux (Stokes) et la déformation élastique de préformes. Une méthode level-set permet de décrire les interfaces fixes ou mobiles de couplage.
\end{abstract}

Mots clés - Stokes - Darcy, matériaux composites, level-set.

\section{Introduction}

Les matériaux composites, obtenus par l'association d'une résine et de renforts fibreux, permettent de produire des structures allégées qui conservent une bonne tenue mécanique. Leur utilisation s'est ainsi répandue dans de nombreux secteurs industriels tels que le transport, le bâtiment, l'aérospatial. Ce travail vise à modéliser et simuler par éléments finis les procédés par infusion de résine utilisés pour élaborer des matériaux composites à matrice organique. Le mélange résine - fibres est réalisé au cours de l'élaboration des structures, réduisant ainsi les coûts de production [1]. Ces procédés, encore mal maitrisés et posant des problèmes de modélisation, peuvent se schématiser comme suit (Figure 1) : le domaine considéré est constitué d'un drainant (milieu perméable, initialement non rempli) et de préformes sèches (milieu poreux, élastique non-linéaire). Sous l'effet d'une pression mécanique extérieure, les préformes sont d'abord compactées. La résine est alors libérée et s'écoule par différentiel de pression, préférentiellement dans le drainant, qualifié alors de milieu purement fluide, avant d'infuser au travers des préformes. Les préformes humides sont alors sujettes à un gonflement, provoqué par la contrainte exercée par la résine.

Cette modélisation fait intervenir un premier problème de mécanique des fluides - milieux poreux, avec un couplage entre un écoulement dans le drainant, régi par les équations de Stokes, et un écoulement dans les préformes, régi les équations de Darcy. Ce problème est abordé dans la Section 2 dans un cadre Eulérien : deux fonctions level-set permettent de réaliser le couplage Stokes - Darcy et résine - air lors de l'écoulement. Le second problème, étudié dans la Section 3 concerne la mécanique des solides, avec les grandes déformations subies par les préformes élastiques non linéaires, puis le gonflement de celles-ci sous l'action de l'arrivée de la résine. Enfin, différentes simulations numériques d'infusion et d'injection de résine sont présentées dans la Section 4.

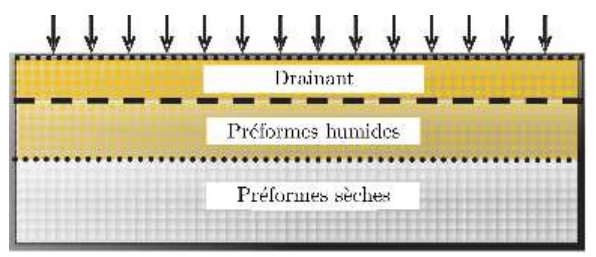

Figure 1 - Modélisation adoptée pour les procédés par infusion de résine : découpage en trois zones (résine seule, préformes humides et préformes sèches). 


\section{Ecoulement de la résine}

\subsection{Modélisation et formulation du problème couplé Stokes - Darcy}

La résine est supposée se comporter comme un fluide Newtonien incompressible, de viscosité $\eta$. Nous supposons ici que l'écoulement est isotherme. En négligeant les effets d'inertie (petit nombre de Reynolds), l'écoulement de la résine dans le drainant $\Omega_{s}$, est décrit par le système de Stokes, formulé en vitesse $v$ et pression $p$ :

$$
\begin{array}{ll}
\nabla \cdot(2 \eta \dot{\boldsymbol{\varepsilon}}(\boldsymbol{v})-p I) & =0 \\
\nabla \cdot \boldsymbol{v} & =0
\end{array}
$$

où $\dot{\varepsilon}(\boldsymbol{v})=\frac{1}{2}\left(\nabla \boldsymbol{v}+{ }^{t} \nabla \boldsymbol{v}\right)$ est le tenseur des vitesses de déformation et $I$ est l'identité. D'autre part, les préformes étant assimilées à un milieu poreux $\Omega_{d}$, l'écoulement de la résine au travers de celles-ci est décrit par les équations de Darcy formulées en vitesse $\boldsymbol{v}$ et pression $p$ :

$$
\begin{array}{ll}
\boldsymbol{v}+\frac{K(\phi)}{\eta} \nabla p & =0 \\
\nabla \cdot \boldsymbol{v} & =0
\end{array}
$$

où $K$ est la perméabilité du milieu, reliée comme nous le verrons à la porosité $\phi$ des préformes.

Le système (1) - (2) est couplé en considérant sur l'interface Stokes - Darcy $\Gamma_{s d}$ la continuité de la vitesse normale, de la contrainte normale et une condition de type Beaver - Joseph - Saffman qui s'écrit [2] :

$$
2 \boldsymbol{n} \dot{\varepsilon}(\boldsymbol{v}) \boldsymbol{\tau}_{j}=-\frac{\alpha}{\sqrt{K}} \boldsymbol{v} \cdot \boldsymbol{\tau}_{j}, \quad j=1,2
$$

où $\boldsymbol{n}$ est la normale unitaire à $\Gamma_{s d},\left(\boldsymbol{n}, \boldsymbol{\tau}_{1}, \boldsymbol{\tau}_{2}\right)$ forme un trièdre direct, et $\alpha$ est un nombre sans dimension correspondant à un coefficient de glissement.

\subsection{Forme faible du problème couplé Stokes - Darcy}

Afin d'effectuer une discrétisation par éléments finis, nous écrivons la formulation variationnelle du problème couplé Stokes - Darcy. Cette formulation s'obtient en sommant les formes faibles duales des problèmes de Stokes et de Darcy, et en prenant en compte les conditions de couplage sur $\Gamma_{s d}$. Voir $[4,3]$ pour plus de détails. Au final, la formulation faible du problème couplé de Stokes - Darcy consiste à trouver $(\boldsymbol{v}, p) \in \mathcal{V} \times \mathcal{P}$ tel que :

$$
\begin{aligned}
\int_{\Omega} H_{s} 2 \eta \dot{\varepsilon}(\boldsymbol{v}): \dot{\varepsilon}(\boldsymbol{w}) d \Omega-\int_{\Omega} p \nabla \cdot \boldsymbol{w} d \Omega & +\sum_{j=1}^{2} \int_{\Gamma_{s d}} \frac{\alpha \eta}{\sqrt{K}}\left(\boldsymbol{v} \cdot \boldsymbol{\tau}_{\boldsymbol{j}}\right)\left(\boldsymbol{w} \cdot \boldsymbol{\tau}_{\boldsymbol{j}}\right) d \Gamma+\int_{\Omega} H_{d} \frac{\eta}{K} \boldsymbol{v} \cdot \boldsymbol{w} d \Omega \\
& =\int_{\Gamma_{N}} p_{\text {ext }} \boldsymbol{w} \cdot \boldsymbol{n} d \Gamma \\
-\int_{\Omega} q \nabla \cdot \boldsymbol{v} d \Omega & =0
\end{aligned}
$$

pour toutes fonctions $\boldsymbol{w}$ et $q$ suffisamment régulières, les composantes de $\boldsymbol{w}$ s'annulant sur le bord $\Gamma_{D}$ où les mêmes composantes de la vitesse sont imposées comme condition de Dirichlet. Le domaine de calcul, $\Omega$, est la réunion de $\Omega_{s}$ et $\Omega_{d}$, tandis que $H_{s}$ et $H_{d}$ désignent les fonctions caractéristiques de ces deux sous-domaines, i.e. les fonctions qui sont égales à 1 respectivement dans la zone de Stokes et dans celle de Darcy, et qui sont nulles ailleurs. De plus, outre la prise en compte des conditions de couplage sur $\Gamma_{s d}$, cette formulation permet d'imposer faiblement une contrainte $p_{e x t} \boldsymbol{n}$ sur une partie de la frontière de $\partial \Omega, \Gamma_{N}$, avec $p_{\text {ext }}$ un scalaire (la pression extérieure) et $\boldsymbol{n}$ la normale unitaire extérieure à $\Omega$. Enfin, nous l'avons dit, les valeurs de la vitesse peuvent être imposées sur une partie du bord $\Gamma_{D}$ via l'espace fonctionnel $\mathcal{V}$. On a alors :

$$
\mathcal{V} \equiv\left\{\boldsymbol{w}: \Omega \mapsto \mathbb{R}^{d}, d=2,3, \boldsymbol{w} \in H_{\Gamma_{D}}^{1}\left(\Omega_{s}\right)^{d} \cap H_{\Gamma_{D}}\left(\operatorname{div}, \Omega_{d}\right)\right\} \quad \text { et } \quad \mathcal{P} \equiv L^{2}(\Omega)
$$

où l'indice " $\Gamma_{D}$ " signifie que la valeur des fonctions de $\mathcal{V}$ (ou de leur composante normale dans $\Omega_{d}$ ) est imposée sur $\Gamma_{D}$. 


\subsection{Discrétisation par éléments finis}

Le système (3) est discrétisé par des éléments finis mixtes. La caractéristique de notre approche est d'être monolithique : le domaine $\Omega$ est discrétisé avec un seul maillage fixe et non structuré, formé de simplexes. L'interface $\Gamma_{s d}$ traverse donc les éléments du maillage. La vitesse et la pression sont approchées par des fonctions continues sur $\Omega$ et linéaires par élément. Il est bien connu que des approximations du même ordre en vitesse et en pression ne sont pas stables, ni pour le système de Stokes, ni pour le système de Darcy. Cependant, les raisons de cette instabilité sont différentes pour ces deux systèmes [5]. Ainsi, deux techniques de stabilisation différentes sont utilisées ici pour stabiliser le système, selon que l'on se trouve dans $\Omega_{s}$ ou dans $\Omega_{d}$. Dans le domaine Stokes, la formulation est stabilisée par l'ajout d'un terme bulle en vitesse (MINI-élément), tandis qu'une stabilisation de type "Hughes variationnal multiscale" est considérée pour Darcy [6]. Cette dernière méthode fait partie des méthodes dites multi-échelles dont le principe repose sur la décomposition des inconnues (ici la vitesse) en une partie résoluble $\boldsymbol{v}_{h}$ et une partie non résoluble $\tilde{\boldsymbol{v}}: \boldsymbol{v}=\boldsymbol{v}_{h}+\tilde{\boldsymbol{v}}$. $\boldsymbol{v}_{h}$ correspond à la solution éléments finis, tandis que $\tilde{\boldsymbol{v}}$ représente les effets aux petites échelles, non captées par le maillage, mais dont la non prise en compte conduit à des oscillations. Ainsi, $\tilde{\boldsymbol{v}}$ est d'abord exprimée en fonction du résidu de l'échelle résoluble. Son approximation fournit alors les termes de stabilisation dans la formulation éléments finis. Notons que les termes issus de ces deux techniques de stabilisation sont présents dans les éléments traversés par l'interface, pondérés par une certaine fraction volumique.

L'interface Stokes - Darcy, est décrite par l'isovaleur zéro d'une fonction distance signée $\alpha_{s d}$ fixe, définie en chaque nœud du maillage. Les valeurs de $\alpha_{s d}$ permettent de savoir si un point $x \in \Omega$ se trouve dans $\Omega_{s}$ ou dans $\Omega_{d}$, et donc de connaître $H_{s}$ et $H_{d}$. De plus, le gradient de $\alpha_{s d}$ permet de calculer la normale à l'interface, et d'en déduire les vecteurs tangents $\tau_{1}$ et $\tau_{2}$ intervenant dans le système (3). Enfin, puisque l'interface $\Gamma_{s d}$ n'est pas définie par un ensemble de faces, mais est définie implicitement par $\alpha_{s d}$, l'intégrale surfacique sur $\Gamma_{s d}$ est transformée en intégrale volumique s'appliquant sur un domaine d'épaisseur $\varepsilon$ de part et d'autre de l'interface. On a alors :

$$
\int_{\Gamma_{s d}} f(x) d \Gamma \approx \int_{\Omega} \frac{1}{\varepsilon} \zeta\left(\frac{\alpha_{s d}}{\varepsilon}\right)\left\|\nabla \alpha_{s d}\right\| f(x) d \Omega \text { avec } \zeta(x)=\left\{\begin{array}{lll}
\frac{1}{2}(1+\cos (\pi x)) & \text { si } & -1 \leq x \leq 1 \\
0 & \text { sinon }
\end{array}\right.
$$

La fonction $f$ est donnée ici par : $f(x)=\frac{\alpha \eta}{\sqrt{K}}(\boldsymbol{v} \cdot \boldsymbol{\tau})(\boldsymbol{w} \cdot \boldsymbol{\tau})$. Les calculs sont effectués en prenant $2 \boldsymbol{\varepsilon}=$ $1,5 h$, où $h$ est la taille de maille.

Enfin, la propagation du front de résine, dans le drainant comme dans les préformes est également décrite grâce à une fonction distance, ou fonction level-set, $\alpha$, continue et linéaire par élément, transportée par la vitesse $\boldsymbol{v}$. Ce transport s'effectue en résolvant l'équation de transport $\frac{\partial \alpha}{\partial t}+\boldsymbol{v} \cdot \nabla \alpha=0$, par une méthode éléments finis stabilisée par une technique SUPG. Afin de conserver la propriété de fonction distance, i.e. $\|\nabla \alpha\|=1$, une étape de réinitialisation est appliquée périodiquement [7]. Enfin, en aval du front de résine, le système (1) - (2) caractérisant l'écoulement est prolongé en considérant l'air comme un fluide Newtonien faiblement visqueux, permettant ainsi d'obtenir un champ de vitesse $\boldsymbol{v}$ continu au travers du front de matière.

\section{Déformation des préformes}

La déformation des préformes, due soit à la pression mécanique appliquée, soit à la pression de la résine, est calculée dans le cadre de la mécanique des solides en grandes transformations, traitée en formulation Lagrangienne réactualisée, en résolvant l'équation donnée par la conservation de la quantité de mouvement et le modèle de Terzaghi :

$$
\nabla \cdot\left(\sigma_{e f f}(\boldsymbol{u})-s p H_{s} I\right)=0
$$

où $s$ est la saturation, prise égale à 1 dans nos calculs, $H_{s}$ est la fonction caractéristique du fluide dans les préformes, $\sigma_{e f f}$ le tenseur de Cauchy donné par la loi de comportement des préformes sèches et $\boldsymbol{u}$ le déplacement. Dans nos simulations, une loi élastique non-linéaire dans le sens transverse au plan des renforts, obtenue depuis des données expérimentales, est utilisée. 
L'évolution de la porosité $\phi$ avec la déformation des préformes découle de l'incompressibilité de chaque fibre et de la conservation de la masse d'un élément de volume fibre. En effet, la masse volumique d'un tel élément s'écrit $\rho=\rho_{f}(1-\phi)$ où $\rho_{f}$ est la masse volumique des fibres. En remplaçant $\rho$ par cette expression dans l'équation de conservation de la masse, et en considérant $\rho_{f}$ comme une constante, nous obtenons :

$$
\frac{\partial \phi}{\partial t}-(1-\phi) \nabla \cdot \boldsymbol{v}_{\text {pref }}=0
$$

où $\boldsymbol{v}_{\text {pref }}$ est la vitesse de déformation des préformes déduite de (4) : $\boldsymbol{v}_{\text {pref }}=\frac{d \boldsymbol{u}}{d t}$. Remarquons que l'équation (5) ne contient pas de terme de convection puisque la déformation des préformes est décrite par une approche Lagrangienne réactualisée : $\phi$ est directement transportée par le maillage. La porosité $\phi$ est approchée par une fonction continue sur $\Omega$ et linéaire par élément, et l'équation (5) est discrétisée par une méthode éléments finis de type Galerkin. Un schéma d'Euler implicite est utilisé pour la discrétisation en temps.

Enfin, la perméabilité $K$ est reliée à la porosité $\phi$ via un modèle de Carman - Kozeny.

\section{Simulations numériques}

Le modèle précédent a été implémenté dans le logiciel Zébulon, développé par le Centre des Matériaux (MinesParitech), l'ONERA, la société Northwest Numerics Software et le centre SMS de l'Ecole des Mines de Saint-Étienne.

\subsection{Simulations d'injection de résine}

Nous présentons d'abord deux cas d'injection de résine, pour lesquels les préformes ne se déforment pas. La figure 2 montre un exemple de simulation 2D d'injection de résine. La ligne noire est l'interface Stokes - Darcy. La résine est représentée en rouge et l'air en bleu. L'écoulement de la résine se fait préférentiellement dans le drainant (Stokes), bien qu'avec la valeur de la perméabilité considérée ici, $K=$ $10^{-8} \mathrm{~m}^{2}$, l'imprégnation de la résine dans les préformes fibreuses se produit bien avant le remplissage complet du drainant (figure 2(b)). D'autre part, le front de résine emprisonne de l'air lorsqu'il atteint le bord gauche du domaine (figure 2(c)). L'air peut éventuellement être chassé en plaçant un évent à cet endroit. Précisons qu'une viscosité égale à 0,03 Pa.s a été utilisée pour la résine.

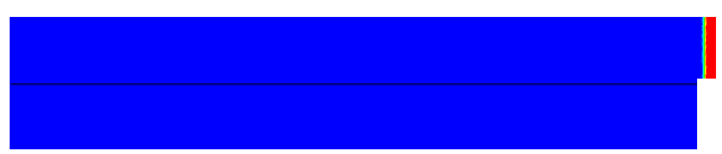

(a)

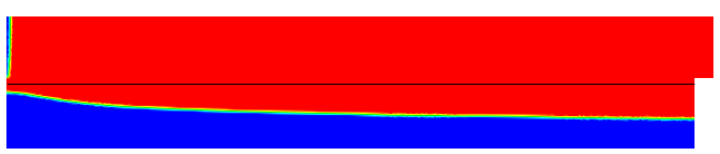

(c)

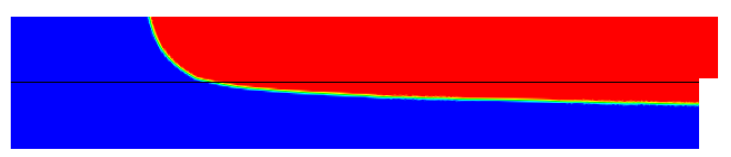

(b)

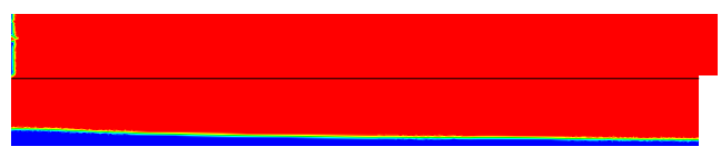

(d)

FIG. 2 - Simulation 2D de l'injection d'une résine avec remplissage du drainant. La résine est représentée en rouge et l'air en bleu.

La Figure 3 montre différentes étapes de la simulation 3D de l'injection de résine dans une pièce coudée. La viscosité et la perméabilité ont les mêmes valeurs que précédemment, et des conclusions analogues peuvent être tirées. Le temps CPU d'une telle simulation, sur un cœur de calcul (processeur 2,2 GHz), est de 1 heure et 20 minutes sur un maillage à 23000 nœuds et 127000 éléments. 


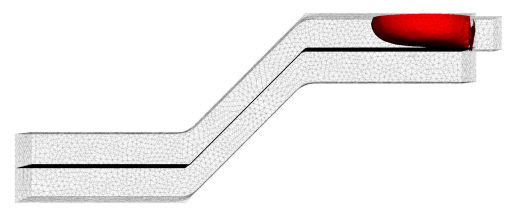

(a)

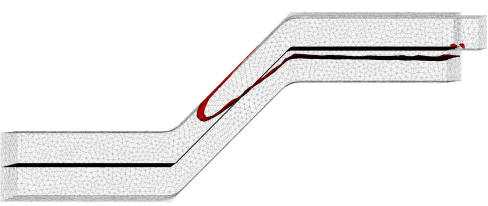

(b)

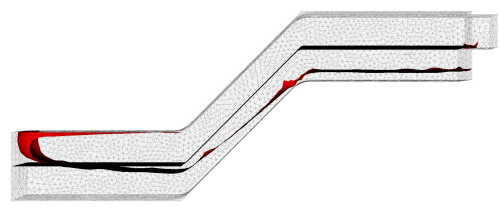

(c)

FIG. 3 - Simulation 3D d'injection de résine.

\subsection{Simulations d'infusion de résine}

Nous présentons à présent une simulation 2D d'infusion de résine prenant en compte la compression puis le gonflement des préformes du à la pression de la résine. Les différentes étapes de cette simulation sont présentées sur la figure 4. La viscosité de la résine est de 0,03 Pa.s, la perméabilité initiale vaut $2,4 \times 10^{-8} \mathrm{~m}^{2}$, tandis que la porosité initiale est de $60 \%$.

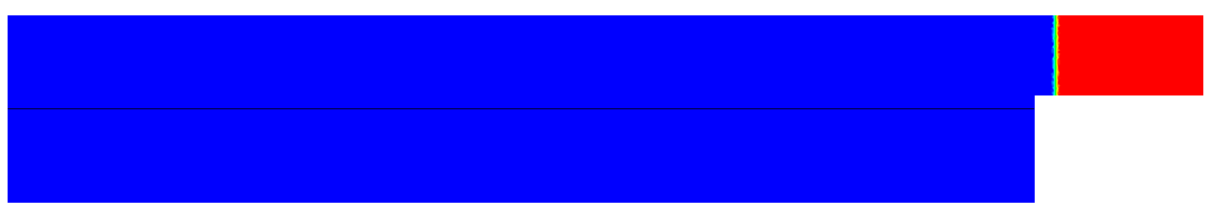

(a)

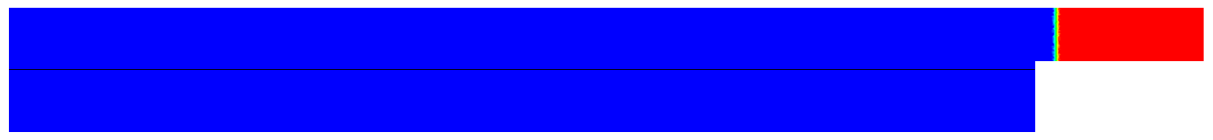

(b)

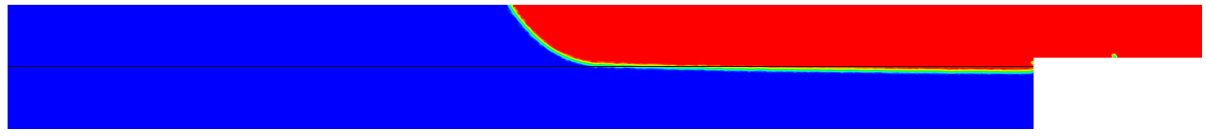

(c)

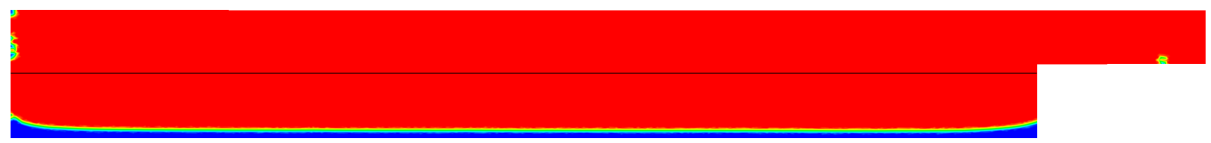

(d)

FIG. 4 - Simulation 2D de l'infusion avec remplissage du drainant.

Une pression de compression de $10^{5} \mathrm{~Pa}$ est appliquée sur le haut du drainant, provoquant le passage de la configuration 4(a) à la configuration 4(b). Tout en maintenant cette pression, la résine est ensuite libérée, et, comme précédemment, s'écoule d'abord préférentiellement dans le drainant. Cependant, la pression que la résine exerce dans les préformes humides provoque un léger gonflement de celles-ci, via le modèle (4), et donc une évolution locale de la porosité suivant l'équation (5). La perméabilité des préformes s'en trouve affectée, modifiant ainsi l'écoulement local de la résine. Le tableau 1 résume les principaux résultats de la simulation. L'évolution de l'épaisseur de l'empilement avec le temps est tracée sur la figure 5 et correspond à la description que l'on vient de faire : on retrouve la phase de compression initiale, suivie de la phase de gonflement.

Pour conclure, nous montrons sur la figure 6 une simulation 3D du procédé d'infusion de résine. Ce cas est en fait l'extension à trois dimensions du cas à deux dimensions. Les mêmes remarques que précédemment peuvent être faites. 


\begin{tabular}{|c|c|c|}
\hline \multicolumn{2}{|c|}{} & $\begin{array}{c}\text { Résultats } \\
\text { numériques }\end{array}$ \\
\hline \hline Valeurs initiales & Epaisseur de l'empilement $(\mathrm{mm})$ & 56 \\
\hline & Fraction volumique de fibres $(\%)$ & 40 \\
\hline \hline Après compression & Epaisseur de l'empilement $(\mathrm{mm})$ & 35 \\
\hline & Fraction volumique de fibres $(\%)$ & 63,73 \\
\hline \hline Après infusion & Epaisseur de l'empilement $(\mathrm{mm})$ & 36 \\
\hline & Gonflement de l'empilement $(\mathrm{mm})$ & 1 \\
\hline & Fraction volumique de fibres $(\%)$ & 62,94 \\
\hline
\end{tabular}

TАВ. 1 - Résultats numériques obtenus pour l'épaisseur de l'empilement et pour la fraction volumique de fibres.

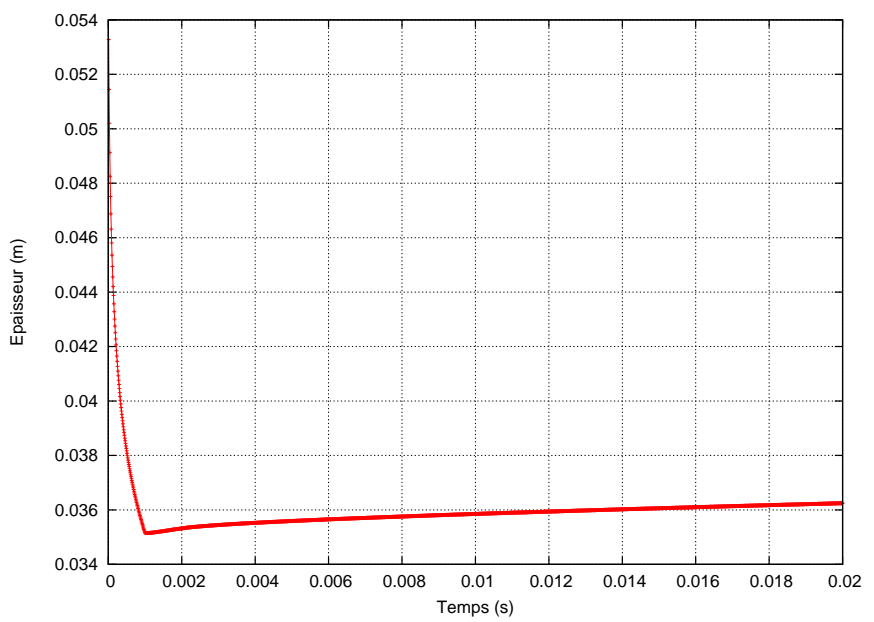

FIG. 5 - Evolution de l'épaisseur de l'empilement en fonction du temps.

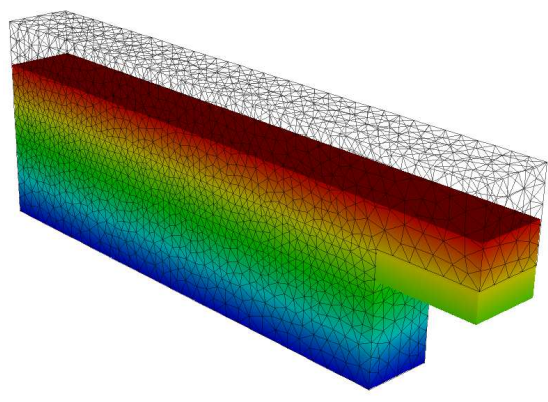

(a)

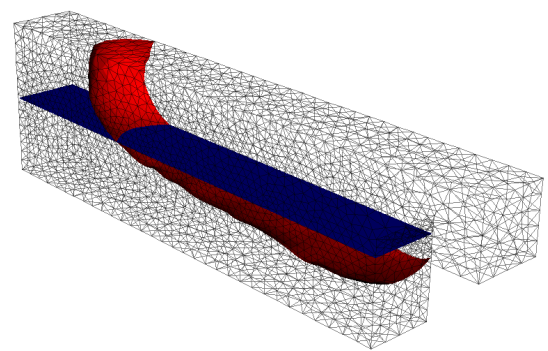

(c)

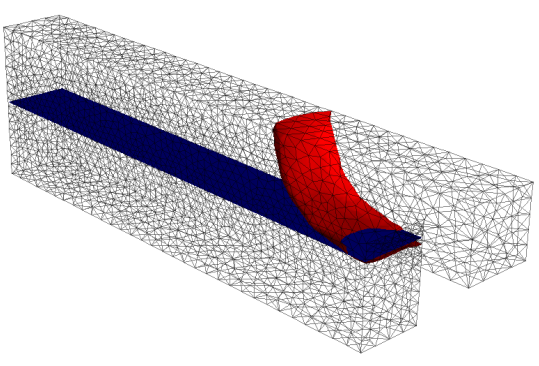

(b)

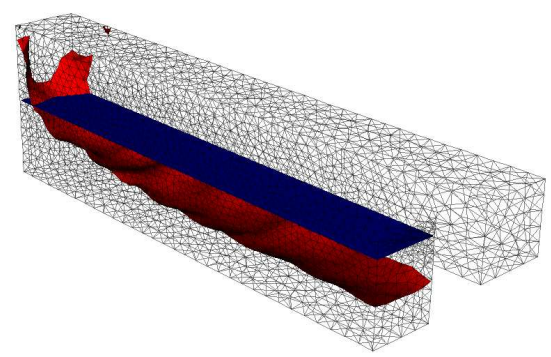

(d)

FIG. 6 - Simulation 3D de l'infusion d'une résine avec remplissage du drainant. L'interface Stokes-Darcy est représentée en bleu tandis que l'interface fluide/air est représentée en rouge. Champ de déplacement après compaction (a), évolution du front de résine $(b) \rightarrow(d)$. 


\section{Conclusion}

Nous avons présenté une stratégie numérique pour la simulation du procédé d'infusion de résine au travers de préformes fibreuses. Cette approche est monolithique, à savoir que tout le domaine de calcul est discrétisé par un maillage unique et non structuré. Une fonction distance (approche de type level-set) permet de décrire l'interface couplant les sous-domaines "Stokes" et "Darcy". La formulation mixte en vitesse - pression, décrivant l'écoulement de la résine, est alors discrétisée à l'aide d'éléments finis $P_{1}-P_{1}$ stabilisés. Les préformes fibreuses ont un comportement élastique non linéaire, couplé à l'écoulement de la résine via un modèle de Terzaghi.

Nous avons montré des exemples de simulations d'injection et d'infusion de résine, en deux et trois dimensions, avec différentes formes de pièces injectées ou infusées. Un des avantages de notre méthode est que la définition des domaines Stokes et Darcy est indépendante du maillage. Ceci facilite son utilisation sur des formes de pièce plus compliquées, ou dans des cas 3D.

Cependant, un certains nombre de points bloquants restent à lever. En premier lieu, la méthode de stabilisation du problème couplé Stokes - Darcy, utilisée dans les éléments coupés par l'interface, n'est pas optimale. En particulier, elle devient insuffisante pour des rapports perméabilité sur viscosité inférieurs à $10^{-11}$. Or, ce sont des valeurs souvent atteintes dans les procédés d'infusion de résine. En second lieu, la simulation des cas 3D demande, pour être améliorée, des maillages plus fins et adaptés localement près des interfaces. Pour gérer de tels cas avec Zébulon, il est nécessaire d'avoir recours au calcul parallèle. Enfin, du point de vue de l'enrichissement du modèle physique, une extension naturelle est la prise en compte du couplage thermo-physico-chimique. Ces différents points font l'objet de deux thèses actuellement dans notre laboratoire (thèses de A. Dereims et de L. Abou Orm)

\section{Références}

[1] S.G. Advani, E.M. Sozer. Process Modeling in Composites Manufacturing, Marcel Dekker, Inc. : New York, 2002.

[2] P.G. Saffman. On the boundary condition at the surface of a porous medium, Studies in Applied Mathematics, 93 - 101, 1971.

[3] G. Pacquaut, J. Bruchon, N. Moulin, S. Drapier. Combining a level set method and a mixed stabilized P1/P1 formulation for coupling Stokes-Darcy flows, International Journal for Numerical Methods in Fluids, Wiley, accepté pour publication.

[4] G. Pacquaut. Couplage Stokes/Darcy dans un cadre Level-set en grandes déformations pour la simulation des procédés d'élaboration par infusion de résine. Thèse soutenue à l'École Nationale Supérieure des Mines de Saint-Étienne, 2010.

[5] S. Badia, R. Codina. Unified stabilized finite element formulations for the Stokes and the Darcy problems, SIAM Journal on Numerical Analysis, 1971 - 2000, 2009.

[6] A. Masud, T.J.R. Hughes. A stabilized mixed finite element method for Darcy flow, Computer Methods in Applied Mechanics and Engineering, 4341 - 4370, 2002.

[7] J. A. Sethian. Level Set Methods and Fast Marching Methods, Cambridge University Press, 1999. 